\title{
Design of Multi-parameter Monitoring System for Indoor Air Quality based on Wireless Sensor Network
}

\author{
Bo Chang ${ }^{1, ~ a ~, ~ X i n r o n g ~ Z h a n g ~}{ }^{2, b}$, Jiang Mingxin ${ }^{1, c}$ \\ ${ }^{1}$ Faculty of Electronic Information Engineering, Huaiyin Institute of Technology, Huai’an, Jiangsu, China \\ ${ }^{2}$ Faculty of Automation, Huaiyin Institute of Technology, Huai'an, Jiangsu, China \\ aemail:mmm33534@sohu.com, bemail:nn33@163.com, 'email: jmx@mail.dlut.edu.cn
}

Keywords: air quality, wireless sensor network(WSN), multi parameter monitoring, design

\begin{abstract}
According to the actual demand of safety monitoring of indoor air quality, low power ZigBee wireless communication technology and micro controller chip, this paper designs a kind of air quality monitoring system for routing monitoring function to realize the unified data management and network, to provide the basis for the evaluation and improvement of environmental air quality. The system collects real-time data of $\mathrm{CO}$, formaldehyde, $\mathrm{SO}_{2}$ and benzene in ambient air, and realizes automatic monitoring of ambient air quality. The staff can analyze the ambient air quality through the data stored and displayed at the receiving end, thus providing technical basis and important support for improving the operation of ambient air quality. The experimental results show that the system has stable performance, high data transmission reliability and strong transplantation ability, and can be widely used in other fields for automatic monitoring of environmental parameters.
\end{abstract}

\section{Introduction}

According to the indoor air quality monitoring data, responding appropriately can reduce the threat to the health of people, and it plays a very important role in improving the quality of life and office efficiency [1-2]. Air quality monitoring can provide people with timely air quality data, quantify air pollution, predict air quality trends, and prevent serious pollution events [3]. At present, the wireless sensor network has become a hot research topic at home and abroad, and has made gratifying progress in many fields, such as military defense, environmental monitoring, safety engineering, [4] and agricultural greenhouses, livestock and poultry farms and food processing and [5], has been a relatively successful application. In western developed countries, air pollution monitoring network is becoming more perfect. Most countries have adopted advanced air and pollutant real-time dynamic monitoring system to carry out monitoring and analysis of [6] at different time and space scales.

Considering the design cost, easy maintenance, real-time data and the expansion of monitoring range, this paper uses wireless sensor network to build a system based on embedded wireless monitoring environment air quality. The network node is composed of ZigBee ad hoc network [7], which can realize the data acquisition, transmission and monitoring and management of [8]. It realizes wireless communication between sensor nodes and gateway nodes through network technology. The staff can manage and configure the system through monitoring center, release monitoring task or collect back data. $\mathrm{CO}$, formaldehyde, $\mathrm{SO}_{2}$ and benzene are the main monitoring items in this system. The system has good portability, and it can be applied to other monitoring fields for different application environments and different sensors. The system has the advantages of low node cost, multi point detection, large amount of data acquisition, high accuracy and little impact on the monitoring environment.

\section{Requirements and Overall Design of Monitoring System}

After market research and analysis, system functional requirements are as follows: the data 
acquisition and transmission; real-time monitoring, emergency alarm can be sent; data management system; management system. The system can automatically detect the working status, that is, the system can automatically detect the working status of the whole system. Indoor air quality monitoring objects: the common pollutants in indoor air are CO (carbon monoxide), formaldehyde, $\mathrm{SO}_{2}$ (sulfur dioxide) and benzene. The system consists of three parts: monitoring center computer, gateway node and sensor node. The block diagram of the system is shown in Fig. 1.

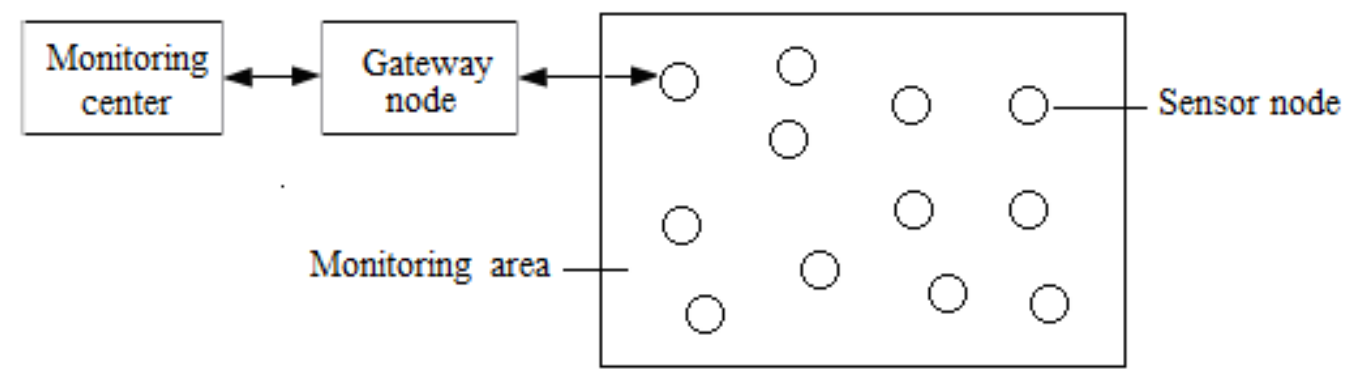

Fig.1. Structure diagram of system

The system realizes the accurate collection of ambient air information by using sensor nodes, in which the data is transmitted wirelessly, and the monitoring data is collected by the multi hop routing to the database of the monitoring center system to store and display the data.

\section{Selection and Design of Hardware Platform for Monitoring System}

\subsection{Design of Monitoring Node.}

The function of monitoring node is to collect, process and transmit the air information. TI wireless communication chip CC2530 is selected as the wireless protocol controller and RF transceiver part, and the chip integrates RF and MCU into one. Considering the specific requirements of the system, development cost, low power consumption and operational reliability and other factors, the node structure includes power supply module, CC2530 wireless transceiver module and gas sensor module. The hardware structure of the node is shown in Fig. 2.

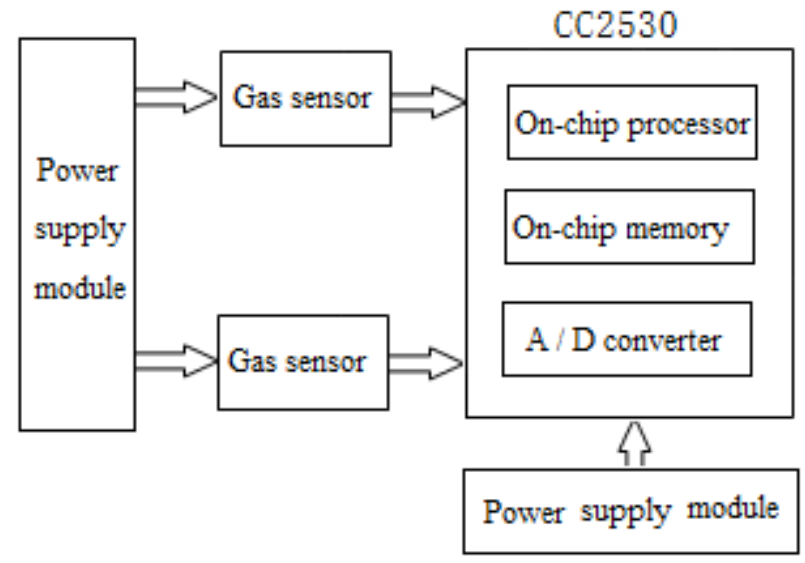

Fig.2. Hardware block of terminal node

\subsection{Hardware Design of Gateway Node.}

Since the main function of the gateway node is to receive, process and transmit the information collected by the sensor node, and considering its connection to the sensor network and the central monitoring computer, storage and communication capabilities are not high for environmental monitoring. Therefore, the gateway node can still be designed with CC2530, which can not only unify the transmission protocol, but also guarantee the reliability of transmission. On the one hand, the gateway can realize receiving data from the sensor network and correction and fusion processing, and sent to computer monitoring center, on the other hand, the gateway can realize sending and receiving center monitoring computer commands, corresponding processing for the 
monitoring center issued instructions, monitoring tasks. Gateway node is mainly composed of wireless receive / send module, embedded processor and memory.

\section{Software Design of Monitoring System}

\subsection{Monitoring Node Software.}

Because the power consumption of nodes in sleep, wake, work and other different modes of work is very different, in the software design, we can combine different work modes organically, which can not only effectively complete the task, but also minimize the power consumption. The main tasks of node software include power self-test, data acquisition module, data receiving and sending and power management. The workflow of a node is shown in Fig.3.

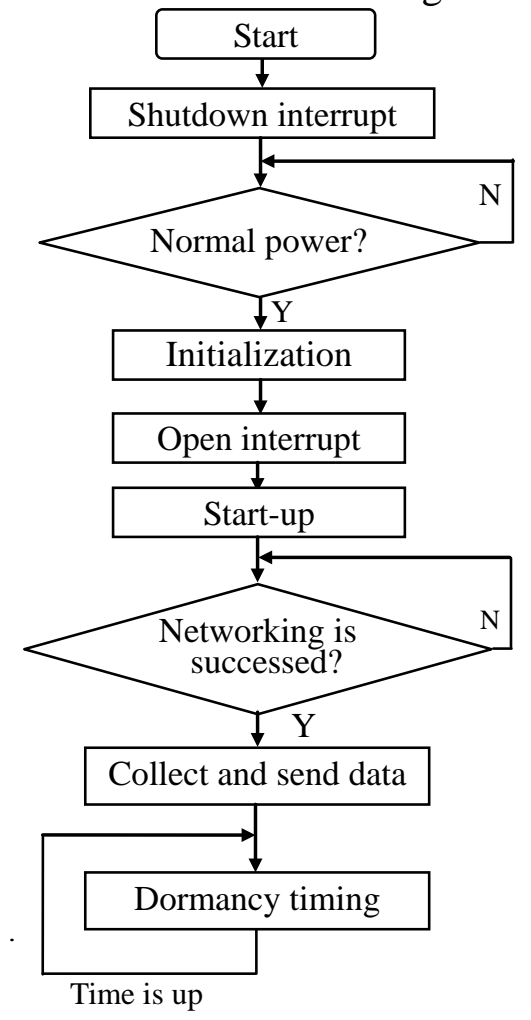

Fig.3. Software flow of the sensor node

\subsection{Gateway Node Software Flow.}

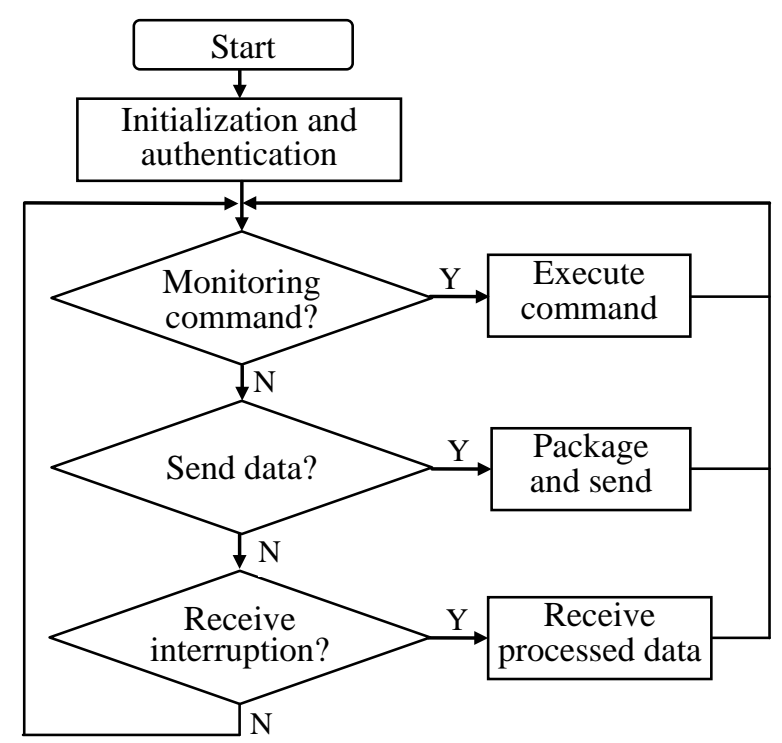

Fig.4. Flow chart of gateway node 
The gateway node is responsible for receiving the data collected by each air sensor node, and then sends the data to the monitoring center after corresponding processing and fusion. In addition, it can also receive the instruction of the monitoring center and adjust the working state of the sensor node. The gateway node workflow is shown in Fig.4.

\subsection{Software of Monitoring Center.}

Monitoring center management system is responsible for sending acquisition commands, receiving acquisition data, while publishing data on the server to meet the client's browsing. The flow chart of program design is shown in Fig.5. Where, after entering the timer, the collected command is written to the serial port queue. An alarm signal will be issued when the number of times $n$ of continuous check is greater than 30. The main tasks of monitoring center include the following:

- Monitor all Flowmeters synchronously to realize on-line monitoring function such as exceeding standard alarm.

- Collect the data and carry out the cyclic redundancy (CRC) check to ensure the reliability of the data.

-Wireless transmission mode, real-time data transmission to the monitoring center.

- Monitoring data and time information can be automatically stored to the database server to realize real-time curve display, history curve query, and data report printing and real-time display according to needs.

- When the data collected by a node is abnormal, record and alarm to eliminate hidden danger.

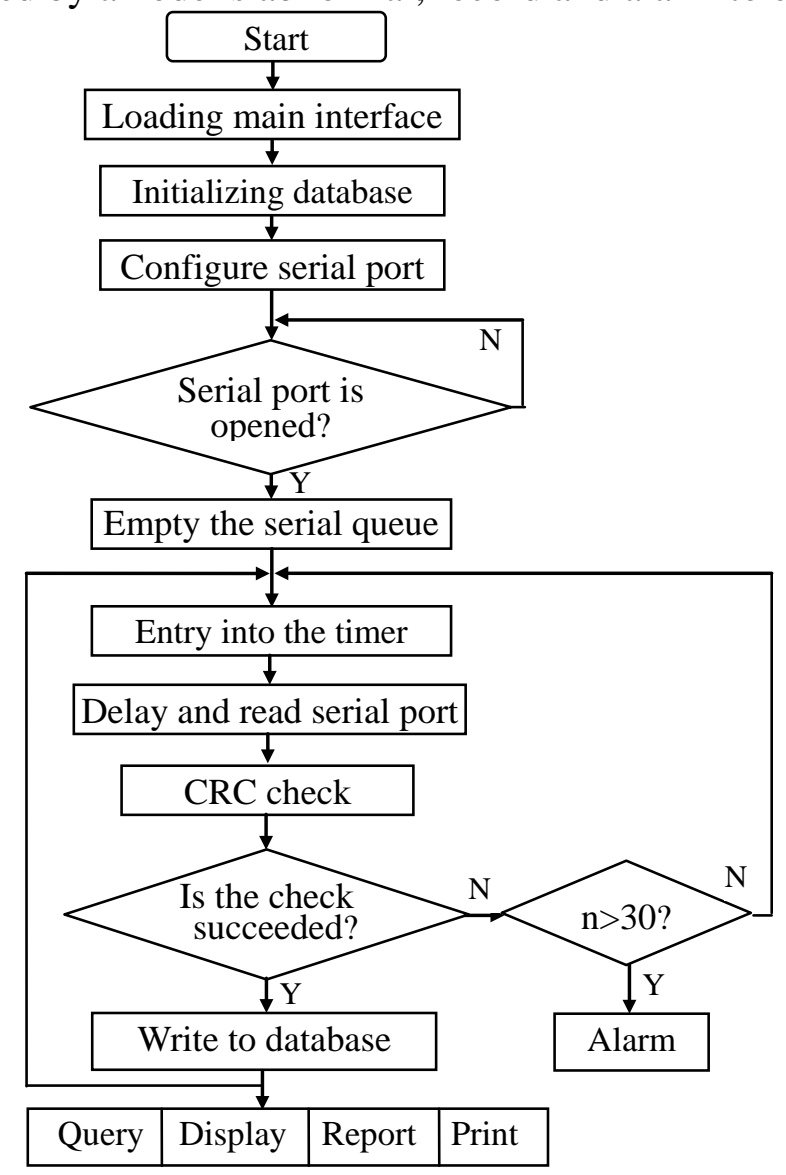

Fig.5. Flow chart of monitoring center

\section{Monitoring Results and Data Analysis}

\subsection{Sensor Selection and Test Environment.}

In this paper, electrochemical CO sensor NAP505 is selected. The detection concentration is 0 $\sim 1000 \mathrm{ppm}$, the precision is less than 5ppm, and the output current is $40+\mathrm{nA} / \mathrm{ppm}$. The 
electrochemical sensor MQ138 is used for formaldehyde and benzene sensors. The detection concentration is $5 \sim 5000 \mathrm{ppm}$, and the precision is less than $+6 \mathrm{ppm}$. We selected electrochemical $\mathrm{SO}_{2}$ sensor $\mathrm{SO}_{2} / \mathrm{CF}-100$, its detection concentration was $0 \sim 20 \mathrm{ppm}$, accuracy less than $+2 \mathrm{ppm}$. According to the system design requirements, we assemble five nodes, in which an central nodes are arranged on the platform, and the remaining 4 sensor nodes are evenly arranged in the approximately $60 \mathrm{~m}^{2}$ laboratory. According to the detection and transmission of ambient air quality parameters, we evaluate the information monitoring function of the system.

\subsection{Test Results and Analysis.}

We first test the transmission distance. In the absence of obstacles, the linear transmission distance of the ZigBee device in the $2.4 \mathrm{GHz}$ band is about $30 \mathrm{~m}$, while the transmission distance of the laboratory instrument interference is about $20 \mathrm{~m}$. Secondly, we test the data transmission. In the four sensor nodes evenly arranged in the laboratory, we put all kinds of air quality sensors for measurement. By comparing with the data collected by 4 sensor nodes, we can verify the accuracy and sensitivity of data transmission of wireless nodes. Now we take one of the nodes to monitor the data statistics, as shown in Table 1.

Table 1. Statistics of indoor monitoring data

\begin{tabular}{|l|l|c|c|c|c|c|c|c|c|c|}
\hline \multirow{3}{*}{$\begin{array}{c}\text { Node parameter value } \\
\text { (unit: }\end{array}$} & \multicolumn{8}{|c|}{ Monitoring time } \\
\cline { 2 - 11 } & $8: 00$ & $10: 00$ & $12: 00$ & $14: 00$ & $16: 00$ & $18: 00$ & $20: 00$ & $21: 00$ & $22: 00$ \\
\hline \multirow{2}{*}{$\mathrm{CO}$} & Monitoring value & 5.87 & 5.92 & 5.85 & 5.77 & 5.89 & 5.76 & 5.63 & 5.54 & 5.50 \\
\cline { 2 - 11 } & Measured value & 5.85 & 5.91 & 5.84 & 5.75 & 5.87 & 5.75 & 5.61 & 5.53 & 5.49 \\
\hline \multirow{3}{*}{ Formaldehyde } & Monitoring value & 0.38 & 0.44 & 0.42 & 0.46 & 0.45 & 0.44 & 0.45 & 0.42 & 0.44 \\
\cline { 2 - 11 } & Measured value & 0.37 & 0.44 & 0.41 & 0.45 & 0.44 & 0.44 & 0.43 & 0.41 & 0.43 \\
\hline \multirow{2}{*}{ Benzene } & Monitoring value & 0.06 & 0.07 & 0.08 & 0.09 & 0.08 & 0.07 & 0.08 & 0.09 & 0.09 \\
\cline { 2 - 10 } & Measured value & 0.06 & 0.07 & 0.08 & 0.09 & 0.08 & 0.07 & 0.08 & 0.09 & 0.09 \\
\hline \multirow{2}{*}{$\mathrm{SO}_{2}$} & Monitoring value & 0.05 & 0.06 & 0.08 & 0.07 & 0.08 & 0.08 & 0.07 & 0.07 & 0.06 \\
\cline { 2 - 10 } & Measured value & 0.05 & 0.06 & 0.07 & 0.07 & 0.08 & 0.07 & 0.06 & 0.07 & 0.06 \\
\hline
\end{tabular}

It can be seen from the above experimental data that the system is more accurate in the detection of $\mathrm{CO}$, formaldehyde, benzene and $\mathrm{SO}_{2}$. By comparing with the 4 measured data, we can see that the relative detection error is controlled within 5\%. The curves of $\mathrm{CO}$ and formaldehyde monitoring and measured data are shown in Fig.6 and Fig.7, respectively.

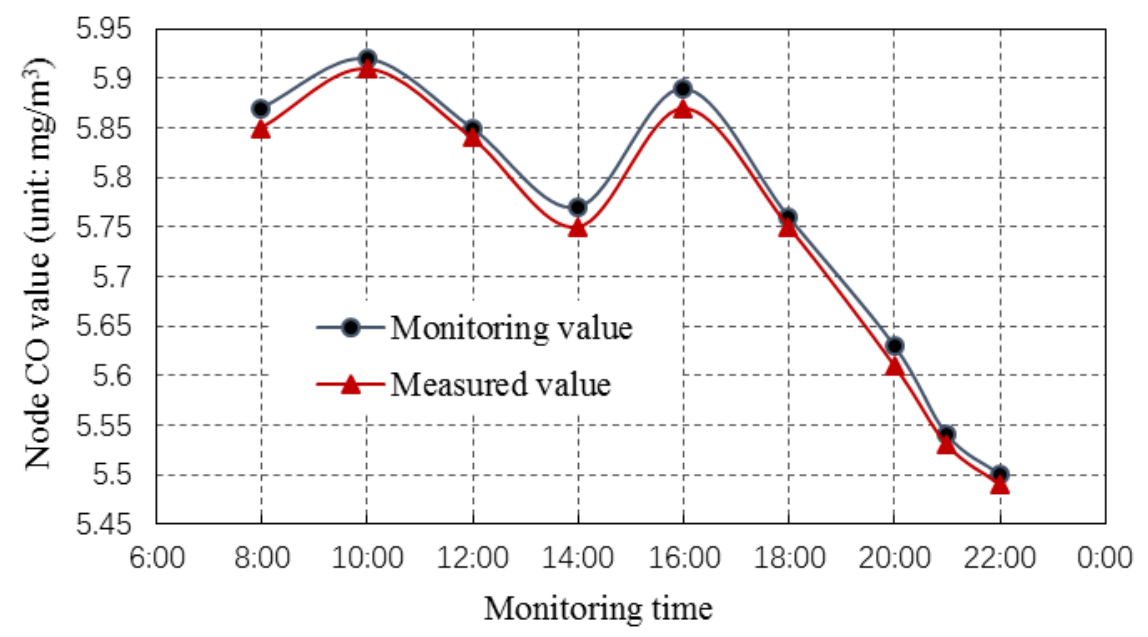

Fig.6. Curve of CO value change 


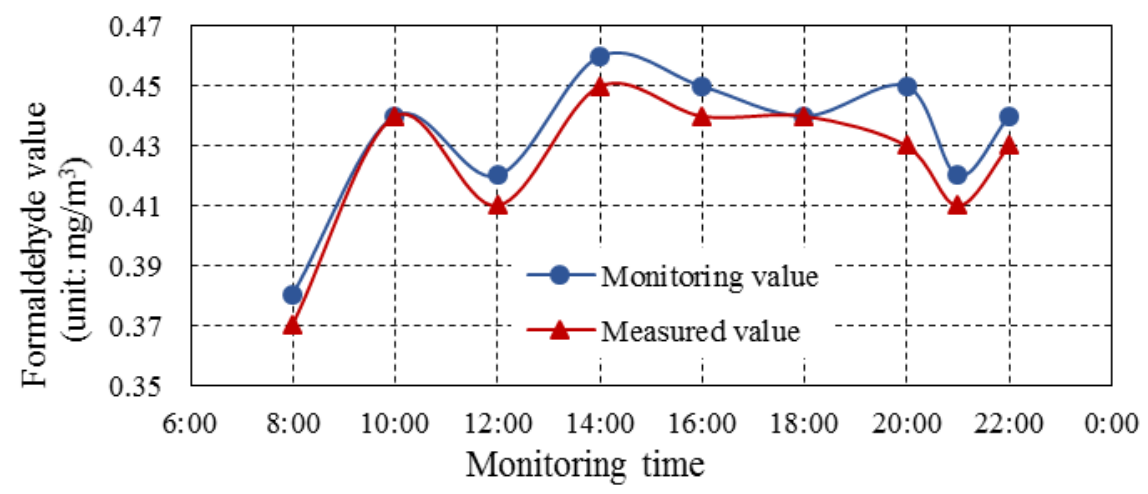

Fig.7. Formaldehyde value change curve

\section{Conclusions}

In view of the problems of network wiring, high cost and poor real-time performance in the current environmental data monitoring, this paper proposes an air quality monitoring system based on wireless sensor networks. We can see that the system can display real-time monitoring point data, can be refreshed at regular intervals, and the historical data displayed in tabular form. By using software method to increase the frequency of data acquisition, the data transmission cycle is prolonged, and the packet loss rate is reduced. Through laboratory testing and data test, the system can reliably monitor the environmental air quality parameters, such as $\mathrm{CO}$, formaldehyde, benzene and $\mathrm{SO}_{2}$. The experimental results show that the system has good applicability, and can achieve accurate data acquisition, transmission and monitoring tasks.

\section{Acknowledgments}

The Natural Science Foundation of Jiangsu Province under Grant BK20171267 supported this work. We would like to thank the anonymous reviewers for their perspicacious comments.

\section{References}

[1] Fabio L.Zucatto. ZigBee for building control wireless sensor networks [J].IEEE International Conference, 2007, (11): 511-515.

[2] Marilena Kampa, Elias Castanas. Human health effects of air pollution [J]. Environmental Pollution, 2008, 151 (2): 362-367.

[3] Exploration on Methods of Historical Environment Protection and Development Based on Concept of "Shan-Shui-City” [J]. China City Planning Review, 2010, 03:50-59.

[4] Sun Zhongfu, Du K M, Han H F, et al. Design of a telemonitoring system for data acquisition of livestock environment[C] //Livestock Environment VIII-Proceedings of the 8th International Symposium, Iguassu Falls, Brazil: ASABE, 2008: 995 1000.

[5] Social Effect of Environmental Pollution on Valley-cities in Western China [J]. Chinese Geographical Science, 2009, 01: 8-16.

[6] Bo LIU, Huiyong ZHENG, Yingzhi LIN. Design of Environmental Monitoring and Control System for Large-scale Pig House with Fermentation Bed [J]. Agricultural Science \& Technology, 2015, 02: 391-399.

[7] Pottie G J, Kaiser W J. Wireless integrated network sensors[J].Communications of the ACM, 2000,43(5): 51-58.

[8] Hill J L, Culler D E. Mica: a wireless platform for deeply embedded networks [J].IEEE Micro, 2002, 22 (6):12-22. 